\title{
ORIGINAL
}

\section{REINCORPORACIÓN AL TRABAJO DESPUÉS DE UN EPISODIO DE INCAPACIDAD TEMPORAL POR CONTINGENCIA COMÚN: PAPEL DE LA EDAD, EL SEXO, LA ACTIVIDAD ECONÓMICA Y LA COMUNIDAD AUTÓNOMA (*)}

\author{
Fernando G. Benavides (1), Manel Plana (2), Consol Serra (1), Ruth Domínguez (1), Miquel \\ Despuig (2), Susana Aguirre (2), Mònica Soria (2), Maite Sampere (1,2) y David Gimeno (3) \\ (1) Unidad de Investigación en Salud Laboral. Departamento de Ciencias Experimentales y de la Salud. \\ Universitat Pompeu Fabra. \\ (2) Mutual Cyclops. \\ (3) Department of Epidemiology and Public Health. University College London. Londres. \\ (*) Proyecto financiado por el Instituto de Salud Carlos III (FIS/041062) y el Ministerio de Trabajo y Asuntos \\ Sociales (FIPROS/78/2007).
}

\section{RESUMEN}

Fundamentos: Describir la duración de episodios incidentes de incapacidad temporal por contingencias comunes según la edad, el sexo, la actividad económica y la Comunidad Autónoma.

Métodos: Incluimos 76.598 episodios de incapacidad temporal iniciados en 2002 en trabajadores afiliados al Régimen General de la Seguridad Social y gestionados por una Mutua de Accidentes de Trabajo y Enfermedad Profesional. La duración mediana y el rango intercuartílico fue estimada por sexo, edad, actividad económica y Comunidad Autónoma. La razón de probabilidad de seguir de baja (RPSB) entre Comunidades Autónomas se ajustó por sexo, edad y actividad económica, tomando Navarra como referencia, mediante un modelo de regresión log-logística con fragilidad gamma compartida.

Resultados: El 25\% de los sujetos habían vuelto al trabajo al $4^{\circ}$ día, el $50 \%$ al $9^{\circ}$ día y el $75 \%$ al $26^{\circ}$ día. Extremadura (RPSB $=2,7$; IC95\%:2,4 a 3,1) y Galicia $(2,6 ; 2,4$ a 2,9$)$ presentaron las diferencias más elevadas respecto a Navarra.

Conclusiones: La reincorporación al trabajo después de un episodio de incapacidad temporal es un proceso complejo que está influido, además de por la edad y el sexo, por la actividad económica y la Comunidad Autónoma.

Palabras claves: salud laboral, seguridad social, absentismo.

Correspondencia:

Fernando G Benavides

Unitat de Recerca de Salud Laboral

Universitat Pompeu Fabra

C/ Doctor Aiguader, 80

08003 Barcelona

\section{ABSTRACT \\ Return to Work After a Non-Work Related Sick Spell: The Role of Age, Sex, Economic Activity and Autonomous Community}

Background: To describe the duration of non-work related sickness absences incidents according to age, sex, economic activity and Autonomous Community.

Methods: The sample of non-work related sick spells included 76,598 incident cases started in 2002 among workers cover by the general regime of the Social Security system, and managed by an insurance company. The median and intercuartils range were estimated by sex, age, economic activities and Autonomous Comunnity. Probability ratio of continuing out of work (PRCOW) were compared among Autonomous Comunities, after adjusting by sexo, age and economic activities, taking Navarra as reference, using a log-logistic regression model with a gamma distribution.

Results: The $25 \%$ of cases there were returned to work at $4^{\circ}$ day, the $50 \%$ at 90 day; and the $75 \%$ at $26^{\circ}$ day. Extremadura (PCOW=2,7; IC95\%: 2,4-3,1) and Galicia $(2,6$; 2,4-2,9) showed the highest differences with Navarra. There were also statistically significant differences among economic activities after adjusting by age and sex.

Conclusions: Return to work after a non-work related sick spell is a complex process, which is influenced by age, sex, economic activities and autonomous community.

Key words: Occupational Health. Social Security. Absenteeism. 


\section{INTRODUCCIÓN}

De acuerdo a la reciente revisión del concepto de discapacidad $^{1}$, la incapacidad laboral puede definirse como una situación dinámica fruto de la interacción entre el estado de salud individual, entendido como bienestar, y las caraterísticas personales y laborales. De acuerdo a la regulación legal ${ }^{2}$, esta situación puede provocar una baja laboral.

Reducir el número de días que un trabajador está de baja como consecuencia de un problema de salud es un objetivo que comparten tanto el trabajador y su empresa como el sistema de salud y el de seguridad social. Pero prevenir el sufrimiento personal, y los costes económicos añadidos, requiere conocer con detalle los factores pronósticos de la duración que sean modificables mediante intervenciones asistenciales y laborales.

En España, cuando un trabajador afiliado al régimen general de la Seguridad Social sufre un problema de salud que le impide llevar a cabo su trabajo y se prevé quese recuperará en los 12 meses siguientes, lo habitual es que su médico le certifique una baja laboral durante el período en que el paciente está recibiendo atención médica. En el caso de que el problema de salud sea de origen laboral, tanto por un accidente de trabajo como por una enfermedad profesional, el médico que certifica la baja será el de su Mutua de accidentes de trabajo y enfermedades profesionales de la Seguridad Social y ésta hará el seguimiento del proceso y abonará la prestación económica correspondiente. Pero si el problema de salud no es laboral, sea por enfermedad común o lesión por accidente no laboral, será un médico del sistema público de salud quien certifique la baja. En este segundo supuesto hablaremos de incapacidad temporal por contingencia común (ITcc).

Desde 1995 las Mutuas, entidades colaboradoras de la seguridad social, tienen también un papel en la gestión de los procesos de ITcc a partir del $16^{\circ}$ día de la baja en aquellas empresas que así lo deciden voluntariamente ${ }^{3}$. De este modo, las $\mathrm{Mu}-$ tuas tienen una posición central para facilitar la adecuada reincorporación al trabajo, después de un episodio de ITcc, mediante intervenciones sanitarias y preventivas.

La literatura reciente muestra que la duración de un episodio de ITcc depende de numerosas variables, algunas de ellas relacionadas con el problema de salud y las características demográficas y sociales del trabajador, pero también con las características de las condiciones de trabajo de su ocupación y la actividad de la empresa ${ }^{4}$.

Con el fin de contribuir a mejorar la gestión de los procesos de ITcc el objetivo de este trabajo fue valorar la relación de la edad, el sexo, la actividad económica y la Comunidad Autónoma con la duración de casos incidentes de ITcc.

\section{MATERIAL Y MÉTODOS}

\section{Población de estudio y definición de casos}

La población base del estudio la constituyen 338.226 trabajadores de las 56.099 empresas afiliadas a una Mutua de ámbito estatal para la gestión de la ITcc. Los sujetos de estudio fueron los trabajadores afiliados al Régimen General de la Seguridad Social de estas empresas que iniciaron un episodio de ITcc durante el año 2002 y fueron seguidos hasta su finalización (como máximo 18 meses, que es el límite legal de permanencia en esta situación: 12 meses más 6 meses de prórroga). Los trabajadores afiliados a los regímenes agrícolas, mar, minas de carbón y autónomos fueron excluidos del estudio dadas las diferencias de las prestaciones entre estos regímenes y el régimen general. 


\section{Información disponible}

Con los datos anonimizados del registro de la Mutua fue posible disponer para cada episodio de ITcc del sexo y la edad (que se agrupó en tertiles) de la persona que estaba de baja, la actividad económica de la empresa (agrupada en los 11 sectores básicos de la Clasificación Nacional de Actividades Económicas), la provincia (agrupada por Comunidades Autónomas -CCAA) y el motivo del alta, además de las fechas de baja y de alta, lo que permitió calcular la duración de cada episodio en días. No fue posible incluir el diagnóstico médico, ya que sólo estaba disponible en el 25,2\% de los episodios.

\section{Análisis}

Para calcular la duración de cada episodio de ITcc iniciado en 2002 se siguió hasta su finalización, ya fuera por curación $(n=50.717)$, mejoría (24.181), alta por la Inspección Médica (1.487) o agotamiento del plazo (213), independientemente de que ello ocurriera con posterioridad al 2002.

Dado que la distribución de los días de duración de los episodios de ITcc no mostraba una distribución normal (estadístico de Kolmogorov-Smirnov = 89,74, p<0,001), se calculó la duración mediana (DM) y el rango intercuartil (RI) correspondiente a los percentiles 25 (P25) y 75 (P75), que informan de los días transcurridos hasta el alta del $25 \%$ y del $75 \%$ de los episodios de ITcc. La DM fue estimada en días para cada variable, utilizando el estimador propuesto por Wang-Chang, que tiene en cuenta que un individuo puede tener más de un episodio $^{5,6}$. Estos cálculos se realizaron con el programa R (survrec funtion).

Asimismo, mediante un modelo de regresión log-logístico con fragilidad gamma compartida $^{7,8}$ se calculó la razón de proba- bilidad de seguir de baja (RPSB) y su intervalo de confianza al 95\% (IC95\%) para cada Comunidad Autónoma, comparándolas con Navarra que presentaba la DM más baja, ajustando por edad, sexo y actividad económica. También se estimo la RPSB y su IC95\% para cada actividad económica, comparándola con la de industria y energía (CNAE: $15-37,40,41)$ que presentaba la DM más baja, ajustando por edad, sexo y Comunidad Autónoma. Estos análisis se realizaron sobre una submuestra de 49.957 episodios de ITcc, una vez excluidos los episodios de ITcc con datos incompletos: en $4.109(5,4 \%)$ no constaba el sexo, en 22.358 $(29,1 \%)$ no constaba la edad, en $349(0,5 \%)$ no constaba la actividad económica y en $107(0,1 \%)$ no constaba la Comunidad Autónoma. Para realizar este análisis fue utilizado el programa STATA.

\section{RESULTADOS}

Durante el año 2002 la Mutua registró 76.598 episodios nuevos de ITcc, lo que representa 2.435.391 días de ausencia del trabajo. Estos episodios tuvieron lugar en 63.397 trabajadores, por lo que hubo $13.201(17,2 \%)$ episodios repetidos al menos dos veces en un mismo trabajador durante este período.

La incidencia en 2002 de episodios de ITcc en el conjunto de la muestra estudiada fue de 22,6 por cada 100 trabajadores. Para el primer episodio, excluyendo los segundos episodios, fue de 18,7 por cada 100 trabajadores.

La DM para el conjunto de episodios fue de 9 días, con un RI entre 4 y 26 días (tabla 1), siendo mayor entre las mujeres ( $\mathrm{DM}=11$ días y $\mathrm{RI}$ entre 5 y 35 días), las personas mayores de 40 años $(\mathrm{DM}=18$ días; RI: 7 y 56 días), para las que trabajaban en la hostelería (DM = 13 días; RI entre 5 y 40 días) y las que vivían en Asturias ( $\mathrm{DM}=13$ días; $\mathrm{RI}$ entre 5 y 45 días). 
Tabla 1

Duración mediana (DM) y rango intercuartil (RI) de la reincorporación al trabajo después de un episodio de incapacidad temporal por contingencia común en trabajadores del Régimen General de la Seguridad Social según el sexo, la edad, la actividad económica y Comunidad Autónoma

\begin{tabular}{|c|c|c|c|c|c|c|}
\hline \multirow[b]{2}{*}{ Variables } & \multirow[b]{2}{*}{ Días } & \multirow[b]{2}{*}{ Episodios } & \multirow[b]{2}{*}{$(\%)$} & \multirow{2}{*}{$\begin{array}{l}\text { DM } \\
\text { Días }\end{array}$} & \multicolumn{2}{|c|}{ RI } \\
\hline & & & & & $\begin{array}{c}\text { Percentil } 25 \\
\text { Días }\end{array}$ & $\begin{array}{c}\text { Percentil } 75 \\
\text { Días }\end{array}$ \\
\hline \multicolumn{7}{|l|}{ Sexo (*) } \\
\hline Hombres & 1.221 .168 & 40.727 & $(53,2)$ & 8 & 4 & 25 \\
\hline Mujeres & 1.139 .338 & 31.762 & $(41,5)$ & 11 & 5 & 35 \\
\hline \multicolumn{7}{|l|}{ Edad (en años) $(\dagger)$} \\
\hline$<=30$ & 664.329 & 22.358 & $(29,2)$ & 9 & 4 & 30 \\
\hline$>30 \mathrm{a}<=40$ & 568.971 & 15.446 & $(20,2)$ & 11 & 4 & 37 \\
\hline$>40$ & 878.788 & 16.513 & $(21,6)$ & 18 & 7 & 56 \\
\hline \multicolumn{7}{|l|}{ Actividad económica CNAE-93 (\$) } \\
\hline Agricultura, industrias extractivas $(1,2,5,10-14)$ & 13.186 & 428 & $(0,6)$ & 10 & 4 & 29 \\
\hline Industria manufacturera y energía $(15-37,40,41)$ & 452.942 & 17.330 & $(22,6)$ & 8 & 4 & 22 \\
\hline Construcción (45) & 387.471 & 11.689 & $(15,3)$ & 9 & 4 & 26 \\
\hline Comercio y reparación vehículos motor (50-52) & 375.393 & 11.650 & $(15,2)$ & 10 & 4 & 30 \\
\hline Hostelería (55) & 256.879 & 6.085 & $(7,9)$ & 13 & 5 & 40 \\
\hline Transporte y comunicaciones (60-64) & 85.443 & 2.446 & $(3,2)$ & 10 & 4 & 31 \\
\hline Intermediación financiera (65) & 245.096 & 8.086 & $(10,6)$ & 10 & 4 & 28 \\
\hline Servicios empresariales $(70-72,74)$ & 265.334 & 9.498 & $(12,4)$ & 9 & 4 & 25 \\
\hline Administración pública, defensa y SS (75) & 33.852 & 1.129 & $(1,5)$ & 9 & 4 & 25 \\
\hline Educación $(73,80)$ & 77.729 & 2.166 & $(2,8)$ & 11 & 5 & 35 \\
\hline Actividades sanitarias y sociales $(85,90-93)$ & 197.122 & 5.742 & $(7,5)$ & 10 & 4 & 32 \\
\hline \multicolumn{7}{|l|}{ Comunidad Autónoma (†) } \\
\hline Andalucía & 327.263 & 8.477 & $(11,1)$ & 11 & 4 & 37 \\
\hline Aragón & 55.930 & 2.016 & $(2,6)$ & 7 & 3 & 18 \\
\hline Asturias & 47.541 & 1.275 & $(1,7)$ & 13 & 5 & 45 \\
\hline Baleares & 32.878 & 945 & $(1,2)$ & 11 & 5 & 31 \\
\hline Canarias & 41.464 & 1.366 & $(1,8)$ & 8 & 4 & 22 \\
\hline Cantabria & 38.873 & 1.351 & $(1,8)$ & 10 & 4 & 28 \\
\hline Castilla León & 118.387 & 4.283 & $(5,6)$ & 9 & 4 & 24 \\
\hline Castilla la Mancha & 49.661 & 1.444 & $(1,9)$ & 8 & 4 & 28 \\
\hline Cataluña & 817.182 & 28.390 & $(37,1)$ & 8 & 4 & 22 \\
\hline Extremadura & 80.017 & 1.900 & $(2,5)$ & 12 & 6 & 41 \\
\hline Galicia & 175.841 & 4.290 & $(5,6)$ & 14 & 6 & 40 \\
\hline Madrid & 274.725 & 8.415 & $(11,0)$ & 11 & 5 & 32 \\
\hline Murcia & 32.705 & 927 & $(1,2)$ & 11 & 5 & 33 \\
\hline Navarra & 58.785 & 2.852 & $(3,7)$ & 5 & 2 & 12 \\
\hline La Rioja & 16.382 & 588 & $(0,8)$ & 7 & 4 & 19 \\
\hline Comunidad Valenciana & 116.265 & 3.608 & $(4,7)$ & 10 & 5 & 28 \\
\hline País Vasco & 146.141 & 4.323 & $(5,6)$ & 11 & 5 & 31 \\
\hline Total & 2.435 .391 & 76.598 & $(100,0)$ & 9 & 4 & 26 \\
\hline
\end{tabular}

(*) Para 4.109 (5,4\%) episodios no consta el sexo. (†) para $22.358(29,1 \%)$ no consta la edad. (\$) para 349 (0,5\%) no consta la actividad económica. (†) para $107(0,1 \%)$ no consta la Comunidad Autónoma y se excluyen 41 casos de Ceuta y Melilla.

Por grupos de edad estas diferencias fueron especialmente relevantes cuando observamos el Percentil 75: entre los menores de 30 años fue 30 días frente a los 56 días (26 días de diferencia) entre los mayores de 40 años. En relación al sexo, la vuelta al trabajo fue más rápido entre los hombres que entre las mujeres, con una diferencia en el Percentil 75 de 10 días (25 días en los hombres y 35 días en las mujeres). 
Tabla 2

Razón de probabilidad de seguir de baja (RPSB) e intervalo de confianza al $95 \%$ (IC95\%) en una submuestra de episodios de Incapacidad Temporal por contingencias comunes $(n=49.957)$

\begin{tabular}{|l|c|c|}
\hline Variables & RPSB $^{\mathbf{1}}$ & IC 95\% \\
\hline Actividad económica (CNAE-93) & & \\
\hline Industria (15-37, 40, 41) & 1 & \\
\hline Hostelería (45) & 1,83 & $1,72-1,94$ \\
\hline $\begin{array}{l}\text { Agricultura, pesca e industrias } \\
\text { extractivas (1,2,5, 10-14) }\end{array}$ & 1,38 & $1,15-1,66$ \\
\hline $\begin{array}{l}\text { Actividades sanitarias y sociales } \\
\text { (85, 90-93) }\end{array}$ & 1,37 & $1,29-1,46$ \\
\hline Educación (73, 80) & 1,40 & $1,29-1,53$ \\
\hline Transporte y comunicaciones (73, 80) & 1,26 & $1,25-1,37$ \\
\hline $\begin{array}{l}\text { Comercio y reparación de vehículos } \\
\text { motor (50-52) }\end{array}$ & 1,31 & $1,25-1,37$ \\
\hline Construcción (55) & 1,27 & $1,21-1,33$ \\
\hline Intermediación financiera (65) & 1,25 & $1,19-1,33$ \\
\hline Servicios empresariales (70-72, 74) & 1,17 & $1,10-1,23$ \\
\hline $\begin{array}{l}\text { Administración pública, defensa y } \\
\text { Seguridad Social (75) }\end{array}$ & 1,17 & $1,03-1,32$ \\
\hline Comunidad Autónoma & RPSB & IC 95\% \\
\hline Navarra & 1 & \\
\hline Extremadura & 2,71 & $2,41-3,04$ \\
\hline Galicia & 2,65 & $2,40-2,92$ \\
\hline Asturias & 2,27 & $1,98-2,60$ \\
\hline Andalucía & 2,25 & $2,06-2,46$ \\
\hline Murcia & 2,17 & $1,85-2,54$ \\
\hline Madrid & 1,90 & $1,74-2,08$ \\
\hline País Vasco & 1,89 & $1,72-2,08$ \\
\hline Comunidad Valenciana & 1,82 & $1,64-2,01$ \\
\hline Castilla-La Mancha & 1,80 & $1,58-2,06$ \\
\hline Baleares & 1,76 & $1,51-2,06$ \\
\hline Cantabria & 1,63 & $1,43-1,86$ \\
\hline Castilla y León & 1,56 & $1,41-1,72$ \\
\hline Aragón & 1,47 & $1,30-1,66$ \\
\hline La Rioja & $1,23-1,72$ \\
\hline Cataluña & $1,33-1,56$ \\
\hline Canarias & \\
\hline & & \\
\hline Asto & & \\
\hline
\end{tabular}

1 Ajustado por edad, sexo y CCAA.

2 Ajustado por edad, sexo y actividad económica.

Al representar las distribuciones de probabilidad de volver al trabajo para aquellas actividades económicas que presentan mayor diferencia, como la industria y la hostelería, observamos que la diferencia en el Percentil 75 entre ambas fue de 18 días (22 días para la industria y 40 días para la hostelería). En relación a las CCAA, las diferencias fueron muy marcadas. Por ejemplo, mientras en Asturias hubo que es- perar hasta 45 días para que el $75 \%$ de los episodios fueran dados de alta, en Navarra sólo 12 días. Entre Madrid y Cataluña también se observó una diferencia, aunque menor: en Madrid hubo que esperar hasta 32 días para que el $75 \%$ de los episodios fueran dados de alta frente a los 22 días de Cataluña.

Hay que señalar que al $16^{\circ}$ día ya han vuelto al trabajo dos de cada tres episodios, y a los 365 días, cuando finaliza el primer período máximo de baja, únicamente queda de baja el 1\% (774 casos) de los episodios.

En la submuestra de episodios con datos completos, al comparar la RPSB entre cada Comunidad Autónoma y Navarra, después de ajustar por sexo, edad y actividad económica (tabla 2) los episodios de ITcc ocurridos en Extremadura (RPSB = 2,71, IC95\%:2,41-3,04) o Galicia (RPSB = 2,69 , IC95\%:2,40-2,92) presentan una probabilidad casi 3 veces mayor de continuar de baja que los episodios de ITcc ocurridos en Navarra. Todas las CCAA mostraron diferencias estadísticamente significativas con Navarra.

Igualmente, al analizar las actividades económicas ajustadas por sexo y edad (tabla 2), observamos que en hostelería la probabilidad de seguir de baja respecto a industria y energía fue casi el doble (RPSB=1,95; IC95\%: 1,83-2,07). Estas diferencias se mantuvieron al añadir las CCAA en el ajuste.

\section{DISCUSIÓN}

Hasta donde sabemos este es el primer estudio que analiza la duración hasta la reincorporación al trabajo examinando una muestra amplia de episodios incidentes de ITcc gestionado por una Mutua en un número elevado de empresas distribuidas por todas las CCAA. 
Los resultados muestran con claridad que el $50 \%$ de los episodios han vuelto al trabajo antes de los 9 días, y que sólo un $25 \%$ supera los 26 días de baja. Resultados algo superiores a los que están disponible para la Comunidad Foral de Navarra ${ }^{9}$, la única Comunidad Autónoma que publica sistemáticamente datos sobre ITcc para el conjunto de afiliados, y donde la mediana fue de 7 días y el percentil 75 de 17 días en 1999. Aunque para los 2.852 episodios de nuestra muestra correspondientes a Navarra, la mediana fue de 5 días y el percentil 75 de 12 días. Lo que nos hace pensar que Navarra presenta valores claramente inferiores a la media española.

Estas diferencias observadas entre las CCAA tienen un especial interés desde el punto de vista de la gestión de estos procesos, pues en principio podríamos postular como hipótesis que no deberían observarse tales diferencias, ya que tanto la regulación legal de la ITcc como los procedimientos de gestión por parte de la Mutua son los mismos para todas las CCAA. Sin embargo, estas diferencias son especialmente llamativas entre Navarra, Aragón o La Rioja, por un lado, y Asturias, Extremadura o Galicia, por otro. Diferencias que se mantienen después de ajustar por edad, sexo y, sobre todo, por actividad económica. Una explicación alternativa, que habría que valorar en futuros estudios, sería que hay diferencias en el seguimiento y control de los episodios de ITcc que se llevan a cabo por parte de los diferentes servicios públicos de salud de las CCAA.

Igualmente, hay que destacar las diferencias observadas entre hombres y mujeres, por grupos de edad y para las diferentes actividades económicas estudiadas. Diferencias que son realmente importantes a partir del Percentil 75. La mayor duración de los episodios de ITcc entre las mujeres respecto a los hombres, y entre las personas mayores respecto a las jóvenes son hechos conocidos ${ }^{10,11}$, pero lo son menos cuando comparamos la duración por ITcc por actividad económica.

Estas diferencias entre actividades económicas no son fácilmente explicables, sobre todo después de ajustar por edad, sexo y CCAA. En principio esperaríamos un resultado opuesto, ya que un episodio de ITcc, por su propia definición, no estaría relacionado con las condiciones de trabajo, a las cuales nos aproximamos a través de las actividades económicas. En este sentido, destaca de manera especial la diferencia observada entre la industria y la hostelería, con una duración significativamente más prolongada en la hostelería, ya que dado el mayor contenido manual de muchas de las ocupaciones de la industria, un problema de salud en ésta requeriría un mayor tiempo de recuperación, lo que retardaría la vuelta al trabajo. Desde el punto de vista de la incidencia lo que ocurre es: las ocupaciones manuales presentan una mayor incidencia de episodios de ITcc que los no manuales ${ }^{12,13}$. No obstante, hemos de señalar que la V Encuesta de Condiciones de Trabajo muestra altos porcentajes de exposición percibida a riesgos ergonómicos (manipulación de cargas, bipedestación, posturas forzadas, etc.) por parte de los trabajadores del comercio y la hostelería ${ }^{14}$. De hecho, los datos de Navarra también muestran para los trabajadores de los servicios una mayor probabilidad de continuar de baja cuando se les compara con la duración del conjunto de afiliados ${ }^{9}$.

Una posibilidad para valorar con mayor capacidad explicativa este resultado habría consistido en comparar las actividades económicas por grupos de patologías. Sin embargo, la ausencia de diagnóstico médico para la mayoría de los episodios ha hecho imposible dicha comparación en el estudio. En todo caso, dada la magnitud de algunas de estas diferencias podemos pen- 
sar en una explicación alternativa relacionada con la organización del trabajo y los factores de riesgo de naturaleza psicosocial, ya que sabemos que determinadas ocupaciones con mayor demanda psicológica y menor control sobre las tareas presentan una duración mayor de los episodios de incapacidad laboral ${ }^{15}$. Estos resultados señalan de manera indirecta la importancia de las condiciones de trabajo en la duración de los episodios de ITcc. Hipótesis que habría que valorar en próximos estudios.

La utilización de la mediana y el rango intercuartil, en lugar de la media y su intervalo su confianza ${ }^{16}$, proporciona una información más ajustada a la distribución real de la duración de los episodios de ITcc, por lo que pensamos que éste es el parámetro estadístico adecuado para la descripción de estos procesos. Además, los datos se han analizado teniendo en cuenta que hay sujetos que pueden tener más de un episodio de ITcc durante el período de observación, en nuestro caso el $17,2 \%$. El porcentaje se incrementa en la medida que el período de observación se prolonga ${ }^{17}$. Este hecho debe valorarse siempre que se analicen episodios de ITcc, ya que no todos los trabajadores, por razones todavía poco conocidas, tienen la misma probabilidad de sufrir un episodio de ITcc.

Diversas limitaciones deben ser tenidas en cuenta a la hora de interpretar adecuadamente los resultados de este estudio. En primer lugar, a pesar de la amplia muestra analizada, ésta se limitó a la gestión de una Mutua. Es posible que podamos encontrar resultados diferentes en otras $\mathrm{Mu}-$ tuas. Ello sugiere la necesidad de realizar estudios de este tipo que comparen los resultados entre las diferentes Mutuas. Asimismo, hay que señalar que en el análisis por actividad económica y por CCAA se excluyó el 34,8\% de los episodios por carecer de información sobre alguna de las variables, lo que puede afectar a los resultados. De hecho, estos episodios con datos incompletos tienden a ser de duración más corta: con una mediana de 7 días frente a los 9 días del total de episodios. Esto puede ser debido a que un proceso de larga duración tiene más posibilidad de cumplimentar los datos ya que el seguimiento administrativo es mayor. Confiamos que, dado el elevado porcentaje de episodios con una duración de 7 o menos días $(40,2 \%)$ incluidos en la submuestra analizada, su impacto en los resultados no variará significativamente las conclusiones alcanzadas. En todo caso, una conclusión útil de este estudio nos lleva a sugerir la necesidad de mejorar la calidad de los registros de ITcc.

En segundo lugar, hemos analizado los episodios de ITcc en las personas afiliadas al régimen general, las cuales tienen características legales específicas en cuanto a la regulación de la ITcc. En futuros estudios debería incluirse también a los trabajadores de los regímenes especiales de la seguridad social, especialmente a los trabajadores autónomos. En tercer lugar, hay que señalar la ausencia del diagnóstico médico de los episodios de ITcc en este estudio, por lo que en futuros estudios habría que analizar los datos para grupos diagnósticos específicos, especialmente los episodios de ITcc motivados por enfermedades musculoesquelécticas y mentales ${ }^{18,19}$.

En conclusión, y teniendo en cuenta estas limitaciones, los resultados de este estudio muestran que la reincorporación al trabajo después de un episodio de ITcc es un proceso complejo que está influido por variables sociodemográficas como la edad y el sexo, variables laborales como la actividad económica y variables geográficas como las CCAA. El estudio cada vez más detallado de estas diferencias puede ayudar a mejorar el conocimiento y la gestión de los procesos de ITcc. 


\section{BIBLIOGRAFÍA}

1. Ayuso-Mateos JL, Nieto-Moreno M, Sánchez-Moreno J, Vázquez-Barquero JL. Clasificación Internacional del Funcionamiento, la Discapacidad y la Salud (CIF): aplicabilidad y utilidad en la práctica clínica. Med Clín (Barc). 2006; 126: 461-6.

2. Sala T. La incapacidad temporal para trabajar derivada de enfermedad o accidente. Valencia: Tirant lo Blanch; 2005.

3. Valenzuela E. El marco normativo de las mutuas en la gestión de la ITCC. La mutua. Revista técnica de salud laboral y prevención 2004; 12: 79-91.

4. Andrea H, Beurskens AJ, Metsemakers JF et al. Health problems and psychosocial work environment as predictors of long term sickness absence in employees who visited the occupational physician and/or general practitioner in relation to work: a prospective study. Occup Environ Med 2003;60:295-300.

5. Wang M, Chang S. Nonparametric estimation of a recurrent survival function. J Am Stat Assoc. 1999; 94: 146-53.

6. Gonzalez JR, Peña EA. Estimación no paramétrica de la función de supervivencia para datos con eventos recurrentes. Rev Esp Salud Pública. 2004; 78: 189-99.

7. Barceló MA. Modelos marginales y condicionales en el análisis de supervivencia multivariante. Gac Sanit 2002; 16 (supl): 59-68.

8. Gutierrez RG. On Frailty models in Stata. Stata Corporation. Disponible en: http://www.stata. com/meeting/7uk/gutierrez.pdf. Acceso 1 de diciembre del 2005.

9. Artieda L, Layana E, Lezaun M et al. Diagnóstico de Salud Laboral en Navarra. Pamplona: Gobierno de Navarra; 2001.

10. Brage S, Nygard JF, Tellnes G. The gender gap in musculoskeletal-related long-term sickness ab- sence in Norway. Scand J Soc Med. 1998;26:3443.

11. Steenstra IA, Verbeek JH, Heymans MW, Bongers PM. Pronostic factors for duration of sick leave in patients sick listed with acute low back pain: a systematic review of the literature. Occup Environ Med. 2005;62:851-60.

12. Morikawa Y, Martikainen P, Head J, Marmot M, Ishizaki M, Nakagawa H. A comparison of socioeconomic differences in long-term sickness absence in a Japanese cohort and a Bristish cohort of employed men. Eur J Public Health. 2004;14:413-6.

13. Benavides FG, Benach J, Mira M, Sáez M, Barceló A. Occupational categories and sickness absence certified as attributable to common diseases. Eur J Public Health. 2003; 13: 51-5.

14. Instituto Nacional de Seguridad e Higiene en el Trabajo. V Encuesta Nacional de Condiciones de Trabajo. Madrid: INSHT; 2003.

15. Gimeno D, Amick III BC, Habeck RV, Ossmann $\mathrm{J}$, Katz JN. The role of job strain on return to work after carpal tunnel surgery. Occup Environ Med. 2005; 62: 778-85.

16. Royo Bordonada MA. La duración de la incapacidad laboral y sus factores asociados. Gac Sanit. 1999;13:177-84.

17. Benavides FG, Sáez M, Barceló MA, Serra C, Mira M. Incapacidad temporal: estrategias de análisis. Gac Sanit. 1999; 13: 185-90.

18. Nieuwenhuijsen K, Verbeek JH, de Boer AG, Blonk RW, van Dijk FJ. Supervisory behaviour as a predictor of retorn to work in employees absent from work due to mental health problems. Occup Environ Med. 2004;61:817-23.

19. Burdof A, Naaktgeboren B, Post W, Prognostic factors for musculoskeletal sickness absence and return to work among welders and metal workers. Occup Environ Med. 1998;55:490-5. 